\title{
INVESTIGACIONES
}

\section{La formación de la comprensión auditiva con fines profesionales}

\author{
The Training of Listening Comprehension with Professional Purposes
}

\author{
Adrian Abreus González ${ }^{a}$ \\ ${ }^{a}$ Departamento de Lenguas Extranjeras, \\ Vicedecano de Investigación y Posgrado en la Facultad de Humanidades. \\ Universidad de Cienfuegos "Carlos Rafael Rodríguez”, Cuba. \\ aabreus@ucf.edu.cu
}

\section{RESUMEN}

El artículo aborda las consideraciones en torno al proceso de enseñanza-aprendizaje de la habilidad de comprensión auditiva en función de la interpretación en la formación de intérpretes profesionales. Su objetivo esencial es describir la necesidad y cualidades de un modelo didáctico para el desarrollo de la mencionada habilidad con un fin profesional, o sea, en función de la interpretación. Para ello se empleó durante el estudio la metodología cualitativa, estableciendo las principales regularidades que fueron resultado de la exploración en la práctica de la problemática expuesta, para su posible solución desde la didáctica. Se muestran además las principales valoraciones del autor en torno al fenómeno estudiado y las valoraciones de los expertos alrededor de las cualidades y carácter sistémico del modelo didáctico que se presenta.

Palabras claves: habilidad profesional, modelo didáctico.

\begin{abstract}
The article presents considerations about the teaching-learning process of listening comprehension skills for interpreting purposes in the training of professional interpreters. The main objective is describing the necessity and qualities of a didactic framework for the development of listening comprehension with a professional purpose. In order to carry out the research, the author used the qualitative methodology, which allowed establishing the main regularities that emerged from the practical exploration of the research problem, in order to find a solution from a didactic point of view. The article also presents the author's analyses around the phenomenon studied, and the assessment of the experts who evaluated the didactic framework proposed, taking into account the qualities and systemic character described.
\end{abstract}

Key words: professional skill, didactic framework. 


\section{INTRODUCCIÓN}

La comprensión auditiva es considerada una de las habilidades más necesarias para el empleo de la lengua sin distinción del carácter nativo o extranjero de esta última. Esta habilidad ha sido ampliamente estudiada por los lingüistas y pedagogos (Siegel, 2015), quienes han desarrollado investigaciones para su perfeccionamiento tanto desde los procesos naturales de desarrollo de la misma, como desde el proceso de enseñanzaaprendizaje de las lenguas. En ese sentido, es válido destacar el papel preponderante de la comprensión auditiva para quienes estudian una lengua extranjera, sobre todo para quienes se forman como intérpretes.

La importancia de esta habilidad en dicha formación radica en las posibilidades que brinda para que el proceso de interpretación de una lengua a otra ocurra como parte de la mediación lingüística entre al menos dos personas que hablan lenguas distintas. Así, es reconocido el papel relevante de la comprensión auditiva en reuniones internacionales, evaluaciones, negocios y la comunicación de manera general (Richards y Burns, 2012), eventos en lo que la participación del intérprete es eminentemente necesaria.

Los programas de formación de intérpretes en el mundo varían de acuerdo a los contextos y necesidades de cada región. Según argumentan (Abreus y Carballosa, 2014a), el énfasis en la formación de estos profesionales en Europa radica en la necesidad de reforzar los conocimientos del idioma para optimizar el nivel de empleo del mismo durante la interpretación. Así, se presta atención al desarrollo de la capacidad comunicativa del futuro intérprete, lo que presupone un buen dominio de los idiomas de trabajo desde el punto de vista lingüístico y cultural.

En el continente americano, por su parte, dicha formación en ocasiones no precisa la enseñanza de los nexos interculturales. Por ello, el futuro intérprete se enfoca en el aprendizaje de los aspectos linguiísticos y no es capaz de extrapolar al proceso de interpretación los aspectos extralingüísticos y paralingüísticos que tienen lugar durante la comunicación. En algunas regiones de este propio continente, como es el caso de Cuba, por ejemplo, los intérpretes se forman bajo una visión que les permite servir como mediadores de la comunicación entre hispanohablantes y no hispanohablantes que empleen la lengua extranjera en la que se forman (esencialmente el inglés).

Aunque con más de cuarenta y cinco años de experiencia en la formación de profesionales de la traducción y la interpretación, la constante búsqueda del perfeccionamiento de la didáctica de esta disciplina ha caracterizado a los investigadores cubanos (Abreus, 2019). Así al realizar un análisis de los principales modelos para la enseñanza-aprendizaje de la comprensión auditiva en inglés como lengua extranjera, el autor del presente identificó algunas carencias que permiten reconsiderar desde la didáctica la forma en que se enseña dicha habilidad en la formación de traductores e intérpretes.

En los modelos analizados (Modelos de Park, 2004; Xiaohui, 2005; Barceló, 2010; Barbosa, 2012), tanto desde el punto de vista lingüístico como pedagógico, se tienen en cuenta cómo ocurren los procesos holísticos de conjunto con los atomísticos para que la comprensión tenga lugar. Asimismo, dichos modelos se formulan sobre la base de un esquema del proceso auditivo que incluye la entrada, la formación y confirmación de hipótesis, los efectos cognitivos y los mensajes recibidos. Sin embargo, ninguno de estos enfatiza en el papel de los elementos paralingüísticos como el tono, ritmo, volumen de la voz, timbre y fluidez verbal durante la comprensión auditiva. 
En ese sentido, esta carencia obstaculiza la preparación de los receptores (futuros intérpretes) para la asimilación del sentido del mensaje que se emite en la lengua de partida (L1) y que debe ser trasmitido en una lengua meta (L2) diferente; de modo que se tengan en cuenta las características ilocutivas del discurso oral.

Por otra parte, no se reconoce el beneficio que la comprensión auditiva aporta a la re-expresión del mensaje emitido en una L2 diferente a la L1 en que fue originalmente comunicado durante el proceso de interpretación, toda vez que los modelos analizados se han dirigido a su enseñanza como proceso o como habilidad en la especialización pedagógica. Ante estos hallazgos se procedió a explorar el estado de desarrollo de esta habilidad lingüística en la formación de intérpretes en Cuba.

Este estudio partió de considerar las carencias siguientes: (a) dificultades en las respuestas ante la comunicación oral inmediata que demanda el proceso de interpretación, debido a la deficiente comprensión auditiva del texto oral a interpretar, (b) incomprensión parcial o total de los aspectos extralingüísticos (roles de los interlocutores, lugar donde se encuentran, etc.) y paralingüísticos en beneficio de la interpretación, así como elementos implícitos de carácter sociocultural presentes en el discurso oral, e (c) imprecisiones en el proceso de enseñanza-aprendizaje de la comprensión auditiva en función de la interpretación, toda vez que para su desarrollo no se tienen en cuenta las particularidades que la caracterizan con este fin específico.

Sobre esta base, el objetivo de la investigación radicó en elaborar un modelo didáctico que favorezca el desarrollo de la comprensión auditiva en beneficio de la interpretación en la formación de los intérpretes. En consecuencia con el objetivo general se definieron en la investigación una serie de objetivos específicos que incluyen:

- Identificar la necesidad de desarrollo de la comprensión auditiva en función de la interpretación en el proceso de formación de los intérpretes.

- Delimitar los referentes teóricos sobre la enseñanza-aprendizaje de la comprensión auditiva y su relación con la interpretación en la formación de intérpretes.

- Describir los resultados de las observaciones realizadas durante la aplicación de las acciones correspondientes a cada etapa para la implementación y la evaluación del modelo para el desarrollo de la comprensión auditiva en función de la interpretación en la formación de intérpretes.

La novedad de la investigación radica en la contribución al perfeccionamiento del proceso de enseñanza-aprendizaje de la comprensión auditiva en función de la interpretación $\mathrm{y}$, por consiguiente, a la mejora de la calidad de este modo de actuación en el profesional de la interpretación.

\section{LA COMPRENSIÓN AUDITIVA EN UNA LENGUA EXTRANJERA}

La comprensión auditiva como habilidad lingüística en una lengua extranjera ha estado presente en las clases de idioma por más de cincuenta años (Vandergrift y Goh, 2012). Sin embargo, su enseñanza ha sido influenciada por aquellas particularidades que caracterizan la enseñanza del resto de las habilidades lingüísticas, a saber: comprensión lectora, expresión oral y expresión escrita. 
Aunque en la actualidad existen disímiles investigaciones relacionadas con la metodología de la enseñanza de la comprensión auditiva como habilidad, la mayoría de las investigaciones siguen poniendo énfasis en el perfeccionamiento de las habilidades escritas y de expresión oral. Así, la literatura especializada refiere dicotomías incluso en cuanto a considerar la comprensión auditiva como habilidad o como proceso.

Para los propósitos de la investigación, se asume la comprensión auditiva desde una perspectiva diferente, en tanto se describen sus particularidades en función del desarrollo de una habilidad profesional como la interpretación, específicamente en la formación de intérpretes. Su análisis parte, por tanto, de considerar que su carácter de habilidad está dado por la operacionalidad que le es inherente y que permite la ejecución de acciones coherentemente diseñadas y organizadas; de modo que su sistematización implique no sólo una repetición de dichas acciones, sino además el perfeccionamiento de las mismas.

Sin embargo, el autor de la investigación considera además que el hecho de que la comprensión auditiva ocurra fuera e independientemente de la incidencia educativa otorga a la misma un carácter de proceso que también se reconoce en la misma.

\subsection{CONCEPTUALIZACIÓNDELACOMPRENSIÓNAUDITIVAENFUNCIÓNDELAINTERPRETACIÓN}

La interpretación es entendida como una actividad traductora subsumida en una noción más amplia de la traducción y que contiene características que la distinguen (Pöchhacker, 2010). Así, según este propio autor, la definición genérica tradicional de la interpretación se refiere a la "traducción oral" del discurso.

Si bien al producto de la actividad que implica la interpretación del significado de un texto cualquiera de una lengua de origen a una lengua extranjera se denomina traducción, la interpretación es reconocida, según (Abreus y Cima, 2013, 2016), como la traducción inmediata y presencial del discurso oral. En ese sentido, esta implica mucho más que el simple reconocimiento de los aspectos lingüísticos que influyen en la comprensión del discurso, en tanto presupone la comunicación oral del mensaje en una lengua diferente a la lengua en que fue emitido originalmente.

Para el logro de una adecuada y efectiva interpretación es imprescindible reproducir los mecanismos, técnicas y estrategias de comprensión auditiva que caracterizan a este proceso en la lengua materna. De este modo, se crean los modelos necesarios para representar mentalmente el proceso de re-expresión del contendido lingüístico y extralingüístico del mensaje en una lengua diferente a la lengua de emisión. Este procedimiento ayudará a que este proceso de representación mental sea el resultado de la combinación de la nueva información a la que tiene acceso el intérprete y el conocimiento previo que posee sobre el contenido del discurso oral a interpretar.

En tal sentido, las experiencias anteriores de comprensión auditiva en la lengua materna servirán de base para el establecimiento de asociaciones meta-cognitivas a niveles semejantes (Abreus y Carballosa, 2014a). La interpretación implica el desarrollo de una serie de habilidades; dentro de las que se encuentran: escuchar, comprender y memorizar la información en la lengua de partida, así como traducir mentalmente, comprimir y "editar" el mensaje de la lengua de partida a la lengua de salida, y finalmente verbalizar el mensaje en esta última (Kornakov, 2000).

Así, la formación y desarrollo de la habilidad de comprensión auditiva coadyuva a la apropiación de expresiones fijas, aforismos y vocabulario que les permite a los estudiantes 
enfrentar el proceso de interpretación de manera efectiva en el contexto laboral. Lo anterior justifica que el desarrollo de la habilidad de comprensión auditiva adquiera una connotación diferente, toda vez que el resultado final de la comprensión no se refiere a la reacción ante el contenido del mensaje oral, sino que tributa al desarrollo de habilidades que favorecen la re-expresión de dicho contenido de manera inmediata y en una lengua meta diferente.

Es por ello, que esta habilidad se convierte para el intérprete en un objetivo que trasciende el reconocimiento de los elementos conocidos para convertirse en un acto de desarrollo de la capacidad de interpretar nuevos mensajes que contienen frases y léxico que nunca antes han sido escuchadas, y para las cuales, en dependencia del tipo de interpretación no han tenido preparación previa.

Asumiendo como componentes esenciales de la habilidad de comprensión auditiva en función de la interpretación las acciones determinadas por (Abreus y Carballosa, 2015), referidas a: (a) la descodificación del mensaje oral en la L1, (b) la recodificación del mensaje oral en una L2 diferente, y (c) la re-expresión oral del contenido del mensaje en la L2 de manera inmediata durante la comunicación; pudiera decirse que en el presente estudio, esta se entiende por la habilidad lingüística que presupone percibir, descodificar e interpretar los símbolos orales, recordar su significado y re-expresarlo de forma oral e inmediata en una lengua de salida diferente a la lengua de partida, para lo cual se debe prestar atención a los aspectos lingüísticos y paralingüísticos que se presentan en un contexto sociocultural dado (Abreus 2015, 2019).

Se trata de responder mediante la comunicación oral a las relaciones de interdependencia que existen entre el discurso oral como proceso de interacción lingüística, el acto comunicativo caracterizado por un propósito, meta o finalidad y las circunstancias socioculturales que rodean una situación determinada y sin la cuales no se puede comprender correctamente. En otras palabras, durante la formación y desarrollo de la comprensión auditiva en función de la interpretación, los interlocutores, el lugar donde se encuentran y otros elementos externos a la lengua constituyen aspectos que apoyan y complementan el proceso de interpretación.

En el desarrollo de la comprensión auditiva en función de la interpretación, la memoria juega un papel fundamental. Según (Casañ, 2009), la memoria es la capacidad del sistema central para adquirir, almacenar y recordar información, a la vez que constituye la base del conocimiento y el pensamiento.

En ese sentido, la memoria es requisito indispensable para la comunicación en tanto permite la fijación, retención y reproducción de la información. Como agrega (Gaiyan, 2010), los oyentes deben ser capaces de retener los elementos lingüísticos en la memoria inmediata lo suficientemente como para interpretar la unidad del discurso al que se enfrentan. Ello permite retener la información en cada fase del proceso de percepción, hasta el momento en que dicha información pasa a formar parte de la memoria mediata.

La capacidad de conservación de la información en la memoria mediata, a su vez, posibilita que los estudiantes transiten por las etapas para la enseñanza-aprendizaje de la comprensión auditiva, a saber: pre-audición, audición y post-audición, y que finalmente respondan a la comunicación de la manera más acertada posible.

Durante la pre-audición, los aprendices activan los conocimientos previos que tienen sobre el tema en cuestión, se contextualizan y preparan para lo que van a escuchar. En la audición, por su parte, es donde se tiene contacto directo con el mensaje acústico y se descodifica y re-codifica el mismo, mientras que en la fase post-auditiva se opera con lo 
que ha sido comprendido para responder a la comunicación y finalmente re-expresar el contenido del mensaje oral.

\subsection{MODELO DIDÁCTICO PARA LA COMPRENSIÓN AUDITIVA EN FUNCIÓN DE LA INTERPRETACIÓN}

La teoría que se aborda en la pedagogía en torno a la aplicación de modelos en el proceso de enseñanza-aprendizaje es diversa. Para Gimeno (1981) un modelo constituye una representación de la realidad que supone un alejamiento de la misma. Es, por tanto, una representación conceptual, simbólica e indirecta que se convierte en parcial y selectiva de aspectos de su realidad, a la vez que centra la atención en lo que considera importante y desprecia aquello que no lo es. Para este propio autor, la riqueza del modelo radica no solo en su función estructurada del conocimiento selectivo que elabora sobre la realidad, sino en la propia teoría que debe desarrollar en torno al objeto modelado.

Autores como Ruiz (2003), Sierra (2004), Güelmes (2005) y Reinoso (2007) se refieren a la modelación como una vía o herramienta necesaria para la transformación de la práctica educativa.

Desde esta perspectiva, en lo referido al empleo de modelos didácticos, según (Sigarreta, 2001), estos constituyen una concepción sistemática que, en el plano de la enseñanza y del aprendizaje, estructuran una determinada práctica dentro del proceso docente-educativo, para incidir en la formación integral de la personalidad del estudiante.

Por su parte, (De Armas y Valle, 2011) coinciden con (Sierra, 2004), quien define al modelo didáctico como una construcción teórico-formal que basada en supuestos científicos e ideológicos pretende interpretar la realidad escolar y dirigirla hacia determinados fines educativos. En su concepción, los modelos poseen características distintivas que permiten su viabilidad y aplicabilidad. Dentro de estas se encuentran su carácter abierto o capacidad de interactuar con el medio, flexibilidad o capacidad de adaptarse y acomodarse a diferentes situaciones dentro de un marco o estructura general, su dinamismo o capacidad de establecer diferentes relaciones potencialmente, así como su carácter probabilístico o capacidad de poder actuar con un margen de error o de éxito aceptable que dé confianza a la acción (Sigarreta, 2001).

La estructura del modelo generalmente encierra una fundamentación y justificación de su necesidad, así como la determinación del contexto social en el que se inserta. De igual forma, incluye su representación gráfica y la explicación de sus significados, exigencias, criterios de uso, argumentación y cualidades; sus formas de instrumentación mediante estrategias, metodologías, variantes o recomendaciones y su evaluación (De Armas y Valle, 2011).

A tenor de las consideraciones teóricas expuestas con anterioridad, el autor de la investigación coincide con los criterios de (De Armas y Valle, 2011), lo que permite resumir que el modelo didáctico se entiende como una construcción teórico-metodológica, que reproduce simplificadamente un proceso determinado para su posterior transformación en la práctica educativa. Así, el modelo que se presenta se elabora partiendo de su concepción didáctica, en tanto permite definir las relaciones dialécticas que se dan en el proceso de enseñanza-aprendizaje de la comprensión auditiva en función de la interpretación.

Al mismo tiempo, establece la relación existente entre la forma, el significado y el contenido, así como los aspectos paralingüísticos (ritmo, entonación, acentuación) que 
intervienen en la comunicación oral, los que permiten comprender significados y reexpresarlos en una L2 con la mayor fluidez y precisión posible.

El modelo didáctico para la compresión auditiva en función de la interpretación parte de considerar la comunicación de un mensaje en una L2 diferente a la L1 como característica esencial del desarrollo de esta habilidad. Asimismo, dado su carácter didáctico, en él se establecen las relaciones internas que tienen lugar entre los componentes de la didáctica: objetivo, contenido, método, medios, formas y evaluación.

En ese sentido, el objetivo del modelo es el desarrollo de la habilidad comprensión auditiva en función de la interpretación, como parte del contenido de las asignaturas de Interpretación en la formación del intérprete. Para ello, es necesario tener en cuenta cómo cada estudiante desarrollará el proceso que, bajo la guía de profesor, le permitirá lograr el fin deseado. Así, el método de enseñanza constituye en el modelo la vía mediante la cual se materializan los contenidos relacionados con las dimensiones cognitivo-comunicativa y la sociocultural esencialmente, sin excluir los aspectos ilocutivos y modales del discurso que permitirán la re-expresión del mensaje.

Estos métodos a su vez están matizados por estrategias de comprensión auditiva holísticas y atomísticas que permiten el tránsito sistémico por cada una de las fases para la enseñanza de esta habilidad $\mathrm{y}$, por tanto, están estrechamente vinculadas al orden jerárquico que se les otorga a las configuraciones contenidas en el modelo didáctico. En tal sentido, las fases pre-auditiva, auditiva y post-auditiva quedan expresadas en el modelo como forma de estructuración de los contenidos que responden a los niveles de asimilación de la habilidad modelada, para lo cual debe prestarse atención a la adecuada selección de los medios de enseñanza, en consecuencia con el método y la forma de organización que se asume en la clase de interpretación; generalmente práctica.

Toda vez que la evaluación tiene un carácter continuo que permite la comprobación del resultado del proceso de enseñanza-aprendizaje y se convierte en una guía orientadora de dicho proceso, la habilidad modelada podrá ser evaluada en la medida en que se transite por las configuraciones que se describen, las que son representativas de las fases, subprocesos y acciones que tienen lugar durante la comprensión auditiva y su enseñanza, relacionadas dialécticamente para concretar la realidad.

En el modelo se precisan tres configuraciones didácticas y la interacción existente entre ellas describe la secuencia lógica del desarrollo de la habilidad de comprensión auditiva en función de la interpretación, de modo que el tránsito por cada una de ellas sea ascendente y secuencial, en forma de sistema. Para ello se tienen en cuenta tanto la adecuada conducción del docente como el activo papel del estudiante durante el desarrollo de la habilidad.

Los criterios de determinación de dichas configuraciones empleados durante la investigación fueron esencialmente: (a) el análisis de los componentes y particularidades de la comprensión auditiva en función de la interpretación, (b) que respondieran a la naturaleza del desarrollo de la habilidad que se modela, (c) la objetividad de las interrelaciones que pueden darse entre ellas.

Las configuraciones didácticas del modelo están dadas por: la percepción y descodificación del mensaje en la L1, la memorización y recodificación del mismo, y el ajuste y re-expresión en la L2. La inclusión de estas configuraciones didácticas en el modelo, en particular la primera de estas, permite evitar que durante el proceso de desarrollo de la comprensión auditiva en función de la interpretación se manifiesten fenómenos directamente relacionados con una deficiente percepción y descodificación del mensaje lingüístico. 
Estos fenómenos se relacionan fundamentalmente con la falta de asociación de una forma de expresión que se materializa a través del significado y una forma de contenido que se objetiva en el significante (Abreus y Carballosa 2014b). En ese sentido, la primera de las configuraciones implica desde la didáctica que se enseñe al intérprete a reconocer, identificar y descomponer el discurso oral y los aspectos contextuales que vehiculan dicho discurso en la lengua de partida. Para ello es imprescindible que se analicen los contenidos, estructuras y formas de presentación del discurso, de modo que los estudiantes puedan percibir y descodificar no solo el concepto, sino la imagen acústica que subyace en él.

En el tránsito por la primera configuración juegan un papel fundamental el cumplimiento de las acciones relacionadas con la activación del conocimiento previo del estudiante sobre el contenido del mensaje oral, lo que presupone la fase de pre-audición. Asimismo, el énfasis fundamental desde el punto de vista didáctico está en la implicación de actividades de audición, en las que el estudiante tendrá acceso directo al lenguaje contenido en el mensaje, que tras ser descodificado debe ser memorizado y re-codificado como parte del proceso de comprensión. Esta fase es vital para el desarrollo de la fase posterior, en tanto constituye el momento en que ocurren con más frecuencia los errores de comprensión auditiva, lo cual obstaculiza el tránsito exitoso por el resto de las configuraciones y fases de la comprensión auditiva en función de la interpretación.

Por otra parte, al memorizar el contenido del mensaje y recodificarlo el estudiante será capaz de incrementar su confianza en la capacidad de retención de la información que contiene el discurso. A su vez, este estará en condiciones de concentrarse en lo que escucha y evitar la diversificación de su atención durante la interpretación. Esta configuración es esencial para que el proceso de ajuste y re-expresión tenga lugar en una lengua meta diferente a la lengua de partida original del discurso.

La implicación didáctica de esta configuración durante la comprensión auditiva en función de la interpretación se centra el en desarrollo exitoso de la post-audición, como fase esencial en la que se superponen la comprensión auditiva y la producción. Una buena orientación hacia las acciones que forman parte de la post-audición garantiza que la reexpresión del mensaje en la L2 ocurra casi de forma automática, lo cual es resultado del desarrollo de la habilidad con un carácter cíclico en su tránsito por cada una de las configuraciones didácticas que componen el modelo.

Finalmente, la re-expresión del mensaje en la L2 ocurre cuando el estudiante ha ajustado el contenido del discurso originalmente emitido en una L1 a aquellas estructuras lingüísticas y extralingüísticas que se presentan en la L2 en que debe re-expresarlo. Así, el objetivo esencial de esta etapa está más relacionado con la comunicación de ideas con una dimensión ilocutiva, que a la precisión de la fuerza elocutiva del discurso comprendido o la proporción de información contextual suplementaria.

La enseñanza de la comprensión auditiva asumiendo el ajuste o paráfrasis del discurso ofrece, desde la didáctica, posibilidades al estudiante de sistematizar las acciones relacionadas con la re-expresión. Estas acciones posibilitarán que se realice una interpretación de forma inmediata haciendo uso de los elementos lingüísticos y extralingüísticos que caracterizan al discurso. Asimismo, deberá prestarse atención no solo a los aspectos relacionados con la dimensión cognitivo-comunicativa de la comunicación, sino a aquellos elementos de carácter sociocultural que tienen lugar durante la misma.

A través de las dimensiones cognitivo-comunicativa y la sociocultural se tienen en cuenta los rastros de los esquemas o estructuras cognoscitivas del emisor del 
mensaje original, los que sirven al estudiante para la comprensión del mensaje que está comunicando como portavoz del discurso. Por ello, en el proceso de enseñanza-aprendizaje de la comprensión auditiva en función de la interpretación, el estudiante debe apropiarse de los recursos relacionados con el procesamiento de información, mediante los cuales se comprenden y producen significados, a la vez que debe emplear estrategias holísticas y atomísticas para obtener y evaluar la información en una lengua de partida y re-expresarla en una lengua meta diferente.

La relación entre lengua, sociedad e individuo(s), materializada en la relación cognición-comunicación-contexto sociocultural, pasa a formar parte de los elementos que coadyuvan a una efectiva comprensión del discurso y, por ende, permiten responder a la comunicación de manera efectiva mediante la re-expresión del contenido del mensaje oral en una lengua diferente a la lengua de partida.

El carácter de sistema de las configuraciones y la objetivación de las dimensiones en cada una de estas está dado por las interdependencias que existen entre ellas, en las que no es posible que se dé la re-expresión de un mensaje que no ha sido previamente percibido, descodificado, ajustado y re-codificado, ya sea en una lengua de partida similar o diferente.

En todos los casos, el objetivo comunicativo se logrará en la medida en que el estudiante sea capaz de re-expresar en una L2 el contenido del mensaje auditivo originalmente trasmitido en una L1 diferente, mediante la aplicación de estrategias de comprensión auditiva que le permitan transitar por cada una de las configuraciones que componen el modelo en su carácter sistémico.

\section{METODOLOGÍA}

Para llevar a cabo el proceso investigativo se empleó una metodología esencialmente cualitativa. Además, se emplearon técnicas de análisis cuantitativos de datos que permitieron contrastar o complementar las conclusiones cualitativas obtenidas como resultado de proceso de investigación.

Se estudió la realidad en su contexto natural, que en el caso específico de la investigación se refiere al contexto de enseñanza-aprendizaje de la habilidad estudiada para su modelación. Dicha realidad fue interpretada tal y como sucedió, intentando proveer sentido a los fenómenos de acuerdo con los significados que tienen para las personas implicadas. Para desarrollar la investigación fue necesario la utilización y recogida de datos a través de una variedad de métodos, dentro de los que tiene un carácter preponderante la observación, sobre todo la participante, considerada por algunos autores como (Rodríguez, Gil y García, 2008), como un elemento esencial que confiere estatus de cualitativas a las investigaciones.

En ese sentido, se partió de considerar los métodos y técnicas de investigación que permitieron realizar el estudio exploratorio y posteriormente corroborar la existencia del problema científico (entrevista, observación, análisis de documentos), los que permitieron abordar los aspectos relacionados con el estado del desarrollo del proceso de enseñanza-aprendizaje de la comprensión auditiva en función de la interpretación en cinco universidades cubanas, y contrastar los resultados obtenidos. Asimismo, se analizaron las principales valoraciones e interpretaciones del autor de la investigación en torno a los resultados del diagnóstico del desarrollo de la habilidad. 
El modelo didáctico fue elaborado sobre la base de las necesidades que, tanto en el orden teórico como práctico, surgieron como resultado de dichas interpretaciones; poniendo especial atención a los elementos de carácter didáctico que posibilitan el desarrollo de la habilidad en los estudiantes, y por ende, la transformación de la habilidad modelada en la práctica educativa.

Los resultados incluyen además una valoración por criterio de expertos de las principales cualidades del modelo didáctico, las que permitieron al autor establecer conclusiones sobre la validez teórica del mismo. De igual modo, se describieron las valoraciones e interpretaciones del autor a tenor de los resultados de la aplicación de las acciones para cada una de las etapas y fases para la implementación del modelo didáctico, donde tiene un papel preponderante la observación participante en sesiones de trabajo de la disciplina Traducción e Interpretación y clases de interpretación en la Universidad de Cienfuegos, mediante el establecimiento de unidades de análisis u observación que permitieron describir las interpretaciones expuestas y por tanto, arribar a las conclusiones que se explicitan en el estudio.

\subsection{ESTUDIO EXPLORATORIO Y DE DIAGNÓSTICO DEL ESTADO DEL DESARROLLO DE LA COMPRENSIÓN AUDITIVA EN FUNCIÓN DE LA INTERPRETACIÓN}

Para llevar a cabo el proceso de diagnóstico del estado del desarrollo de la comprensión auditiva en función de la interpretación se aplicaron una serie de instrumentos y técnicas de investigación científica, que permitieron determinar las principales regularidades que se describen en este acápite. Estos instrumentos y técnicas incluyen: la entrevista a profesores de la asignatura Interpretación en las Universidades de Cienfuegos, Matanzas, Camagüey, Holguín y Oriente, así como la observación a clases de Interpretación y a la ejecución de Talleres de Interpretación con los estudiantes de 4to año de la carrera Lengua Inglesa con Segunda Lengua Extranjera en la Universidad de Cienfuegos.

La entrevista aplicada incluyó interrogantes relacionadas con la frecuencia con que los profesores realizan en sus asignaturas tareas relacionadas con el desarrollo de la comprensión auditiva en inglés y la necesidad de su inclusión en el proceso de enseñanzaaprendizaje del idioma, así como los procedimientos que emplean para el logro de este objetivo en función de la interpretación. Además, se recopilaron los criterios de los docentes en torno a la importancia de la exposición a diferentes variantes de la lengua durante la comprensión auditiva y la jerarquización de los principales problemas que presentan los estudiantes para el desarrollo de esta habilidad.

La muestra para la entrevista estuvo conformada por 8 doctores en Ciencias, 16 másteres en áreas relacionadas con la educación y la enseñanza de lenguas, y 19 licenciados. En todos los casos se tuvieron en cuenta la experiencia como profesores de Interpretación, la categoría docente y/o científica, la especialidad de su formación inicial y la superación recibida desde el punto de vista didáctico sobre el desarrollo de la comprensión auditiva como habilidad del idioma.

Por otra parte, se realizaron observación a clases de Interpretación durante el diagnóstico a fin de conocer en la práctica el estado real de la enseñanza-aprendizaje de la comprensión auditiva en función de la interpretación. Se observaron un total de 56 clases de las asignaturas Interpretación I (10), Interpretación II (8), Interpretación III (12), Interpretación IV (8), Interpretación V (8) e Interpretación VI (10) en el Tercer, Cuarto y 
Quinto años de la carrera Lengua Inglesa con Segunda Lengua Extranjera en la Universidad de Cienfuegos. Dichas observaciones fueron realizadas en el curso académico 2016-2017.

Para la realización de la observación se tuvieron en cuenta un total de 12 parámetros, cuyos resultados revelaron que todos los aspectos se encontraban afectados en el proceso de desarrollo de la comprensión auditiva en función de la interpretación.

Estos parámetros fueron: (a) Realización de actividades relacionadas con el desarrollo de la comprensión auditiva en inglés en las que se incluyen ejercicios para el entrenamiento de la memoria a corto y largo plazo,

(b) Realización de actividades de comprensión auditiva dirigidas a la re-expresión del contenido del mensaje en la propia lengua de partida,

(c) Análisis y reflexión acerca del papel de la comprensión auditiva en función de la interpretación en la formación del profesional de la Lengua Inglesa con Segunda Lengua Extranjera,

(d) Reflexión acerca de las divergencias de la comprensión auditiva para el receptor habitual e intérprete a fin de reconocer nuevas etapas para su desarrollo,

(e) Empleo de actividades o tareas auditivas en las que se presta atención a los elementos extralingüísticos y paralingüísticos del lenguaje (ritmo, entonación),

(f) Empleo de actividades auditivas donde se pone énfasis en los aspectos socioculturales característicos de la cultura del emisor, a fin de ser transmitidos en la lengua meta con igual sentido,

(g) Desarrollo de ejercicios de comprensión auditiva prestando énfasis a la re-expresión del mensaje en la lengua de salida,

(h) Realización de actividades dirigidas a distinguir los registros formales e informales que se emplean en la comunicación del mensaje oral objeto de comprensión y re-expresión,

(i) Retroalimentación inmediata al estudiante una vez que concluye la comprensión auditiva y con ella la re-expresión del mensaje oral,

(j) Se tiene en cuenta el grado de conocimiento previo que tiene el estudiante sobre el tema objeto de la comprensión auditiva que debe ser interpretado,

(k) Identificación de las estrategias de audición que pueden emplearse en función de la interpretación y

(1) Entrenamiento al estudiante en la re-expresión del mensaje como parte de la etapa post-auditiva durante el proceso de comprensión.

Se realizó además un análisis del Modelo del Profesional y los programas de la Disciplina Traducción e Interpretación en la formación del profesional de la Lengua Inglesa con Segunda Lengua Extranjera, se tuvo en cuenta una guía para el análisis documental cuyo objetivo fue constatar las potencialidades o limitantes de dichos documentos en torno a la concepción de la enseñanza-aprendizaje de la comprensión auditiva en función de la interpretación y realizar valoraciones al respecto.

Finalmente, se comparó la información obtenida a través de las técnicas y métodos de investigación científica. Ello permitió determinar las regularidades (coincidencias y divergencias) en torno a la enseñanza-aprendizaje de la comprensión auditiva en función de la interpretación, para lo cual se empleó la triangulación.

Las categorías de análisis que se tuvieron en cuenta para triangular los resultados fueron las siguientes: (a) Inclusión de tareas relacionadas con la comprensión auditiva en función de la interpretación, (b) Atención a los elementos extra y paralingüísticos durante la comprensión auditiva en función de la interpretación, (c) Papel de los elementos 
socioculturales durante la comprensión auditiva en función de la interpretación, (d) Empleo de estrategias de comprensión auditiva para el desarrollo de la habilidad en función de la interpretación, (e) Tratamiento didáctico de la habilidad comprensión auditiva en función de la interpretación.

Mediante la triangulación se logró una mayor objetividad del diagnóstico realizado, lo cual condujo a establecer conclusiones más acabadas sobre la habilidad investigada. En todos los casos se contrastaron los criterios en torno al desarrollo de la comprensión auditiva en función de la interpretación con los elementos observados y obtenidos mediante los instrumentos.

Las principales regularidades fueron las siguientes: (a) Necesidad de sistematizar el empleo de tareas relacionadas con la comprensión auditiva en función de la interpretación en las que se preste atención al desarrollo de la memoria, la concentración y el vocabulario en función de la re-expresión del contenido del mensaje de forma inmediata, (b) Necesidad de enfatizar en el reconocimiento y utilización de los elementos extralingüísticos y paralingüísticos del discurso oral durante la comprensión auditiva en función de la interpretación, (c) Insuficiente tratamiento de los aspectos socioculturales para facilitar la re-expresión del mensaje oral durante la comprensión auditiva, (d) Necesidad de aplicar estrategias holísticas y atomísticas durante la comprensión auditiva en función de la interpretación, (e) Insuficiente tratamiento didáctico de la habilidad de compresión auditiva en su adecuado tránsito por las fases pre-auditiva, auditiva y post-auditiva, en función de la interpretación.

La determinación de los elementos anteriores como resultado de la triangulación permitió al autor de la presente reconocer la necesidad de desarrollar un proceso de enseñanzaaprendizaje de la comprensión auditiva en función de la interpretación caracterizado por una concepción sistémica. En ese sentido, juegan un papel fundamental los mecanismos de percepción, descodificación, memorización y re-expresión de los mensajes orales, lo cual posibilita transformar la formación del estudiante en torno al desarrollo de la comprensión auditiva con un fin profesional, en función de la interpretación.

\subsection{VALORACIÓN TEÓRICA DE LAS CUALIDADES DEL MODELO POR CRITERIO DE EXPERTOS}

Para evaluar la pertinencia del modelo didáctico se empleó el Método Delphi como método de consulta a expertos, en tanto este permite tomar en cuenta la experiencia y conocimientos de estas personas en torno a una temática determinada. Se asumió como expertos a individuos capaces de ofrecer valoraciones conclusivas del problema en cuestión y hacer recomendaciones respecto a sus momentos fundamentales con un máximo de competencia.

El cuestionario de valoración se envió a los expertos para la determinación de su coeficiente de competencia $(K)$, calculado mediante la fórmula: $K=1 / 2(k c+k a)$, donde $k c$ es el coeficiente de conocimiento que tiene el experto sobre los aspectos metodológicos para el desarrollo de la comprensión auditiva como habilidad del idioma inglés, calculado mediante la valoración del propio experto en torno a su conocimiento sobre la temática. Para realizar dicha valoración, el experto seleccionado debió distinguir en una escala de 0 a 10 su nivel de conocimiento en torno a la temática a evaluar, el cual es posteriormente multiplicado por 0,1 para obtener finalmente el valor de dicho coeficiente.

Por otra parte, $k a$ se refiere al coeficiente de argumentación o fundamentación, para lo cual se brindó a los expertos una tabla patrón que permitiera determinar el grado de 
influencia de cada una de las fuentes de argumentación proporcionadas según sus criterios.

El resultado final del $k a$ de cada experto se calculó mediante la suma de cada uno de los valores que los expertos refirieron en la tabla patrón. Para ello se empleó la fórmula: $k a=F 1+F 2+F 3+F 4+F 5+F 6$, donde $F$ es el valor correspondiente a cada una de las fuentes de argumentación presentadas en la tabla patrón.

Una vez obtenidos los valores de $k c$ y $k a$ de cada uno de los expertos, se procedió a calcular el coeficiente $K$ de cada uno de estos, a fin de determinar el coeficiente de competencia del listado final de expertos. Para la interpretación de dichos resultados se tuvo en cuenta el siguiente código: si el resultado oscila entre $0,8<K<1,0$ el coeficiente de competencia es Alto; si el resultado oscila entre $0,5<K<0,8$ el coeficiente de competencia es Medio y si el resultado es $K<0,5$ el coeficiente de competencia es Bajo.

Una vez aplicado el método Delphi para hallar la concordancia entre los expertos, y determinado el coeficiente de competencia $K$ que fundamenta la consideración de cada uno de ellos para trabajar en esta investigación, se obtuvo como resultado que el 90,48 \% de los expertos posee una competencia Alta y el 9,52\% posee un nivel de competencia Media.

Tabla 1. Nivel de competencia $\mathrm{K}$ de los expertos que evaluaron el modelo didáctico

\begin{tabular}{|ccc|}
\hline Experto No. & índice & $\begin{array}{c}\text { Nivel de } \\
\text { Competitividad }\end{array}$ \\
\hline $\mathbf{1}$ & 0,9325 & Competencia Alta \\
\hline $\mathbf{2}$ & 0,9825 & Competencia Alta \\
\hline $\mathbf{3}$ & 0,99 & Competencia Alta \\
\hline $\mathbf{4}$ & 0,9825 & Competencia Alta \\
\hline $\mathbf{5}$ & 0,79 & Competencia Media \\
\hline $\mathbf{6}$ & 0.99 & Competencia Alta \\
\hline $\mathbf{7}$ & 0,8025 & Competencia Alta \\
\hline $\mathbf{8}$ & 0,8425 & Competencia Alta \\
\hline $\mathbf{9}$ & 0,8825 & Competencia Alta \\
\hline $\mathbf{1 0}$ & 0,91 & Competencia Alta \\
\hline $\mathbf{1 1}$ & 0,86 & Competencia Alta \\
\hline $\mathbf{1 2}$ & 0,99 & Competencia Alta \\
\hline $\mathbf{1 3}$ & 0,94 & Competencia Alta \\
\hline $\mathbf{1 4}$ & 0,99 & Competencia Alta \\
\hline $\mathbf{1 5}$ & 0,815 & Competencia Alta \\
\hline $\mathbf{1 6}$ & 0,995 & Competencia Alta \\
\hline $\mathbf{1 7}$ & 0,7475 & Competencia Media \\
\hline $\mathbf{1 8}$ & 0,9025 & Competencia Alta \\
\hline $\mathbf{1 9}$ & 0,86 & Competencia Alta \\
\hline $\mathbf{2 0}$ & 0,8025 & Competencia Alta \\
\hline $\mathbf{2 1}$ & 0,8025 & Competencia Alta \\
\hline & & \\
\hline
\end{tabular}

Fuente. Elaboración propia. 
Sobre la base de estos resultados, se determinó la utilización de 21 expertos a los efectos de considerar sus criterios para la valoración teórica que se propone en esta investigación.

Los resultados del procesamiento estadístico de las respuestas dadas por los expertos muestran que las cualidades del modelo didáctico y sus interrelaciones son Muy Adecuados. Así, las valoraciones realizadas por los expertos evidencian la factibilidad de la concepción teórica del modelo didáctico para la comprensión auditiva en función de la interpretación y la pertinencia de los fundamentos.

Los expertos destacan la flexibilidad de las acciones que conforman las etapas para la implementación y la evaluación del modelo didáctico, de modo que estas pueden ser aplicadas en contextos diversos de formación de los licenciados en Interpretación. Asimismo, estas se pueden adaptar de acuerdo a los niveles de complejidad del contenido de las asignaturas de Interpretación en dicha formación.

De manera general, existió consenso en cuanto a la pertinencia de los criterios de implementación y evaluación del modelo didáctico propuesto y su aplicabilidad en la formación del Licenciado en Lengua Inglesa con Segunda Lengua Extranjera.

\section{DISCUSIÓN Y CONCLUSIONES}

Para realizar la implementación práctica de las acciones que posibilita la materialización del modelo didáctico se tuvo en cuenta la realización de observaciones participantes a sesiones de trabajo del colectivo de la Disciplina Traducción e Interpretación y a clases de Interpretación I, II, III, IV, V y VI en los grupos de tercero, cuarto y quinto años de la carrera Lengua Inglesa con Segunda Lengua Extranjera en el curso escolar 2016-2017. La edad promedio de los estudiantes implicados en el estudio es de 21-23 años. Dichas observaciones se desarrollaron en la Universidad de Cienfuegos.

En las sesiones de trabajo observadas, se confirmó el valor que representan las acciones correspondientes a la etapa de familiarización para la posterior implementación del resto de las etapas que viabilizan la materialización del modelo en la praxis. Además, se tuvieron en cuenta las reflexiones de los participantes sobre el papel de la memoria a corto y largo plazo en la recodificación, ajuste y re-expresión del mensaje como parte de la comprensión auditiva en función de la interpretación.

Las principales valoraciones relacionadas con los factores asociados al desarrollo de la comprensión auditiva en función de la interpretación que se pudieron observar fueron: (a) La presencia de mecanismos extra y paralingüísticos durante el acto comunicativo que se comprende y re-expresa, (b) La presencia de múltiples contextos en los que ocurre la comunicación, (c) La variedad de acentos, ritmos y entonaciones en dependencia de la región o variante lingüística que emplea el emisor del mensaje que se comprende, (d) La pluralidad de elementos socioculturales presentes en el discurso, (e) Los registros del lenguaje que se emplean durante la comunicación por parte del emisor, (f) La marcada importancia de la memoria a corto y largo plazo para una adecuada comprensión auditiva y posterior re-expresión del mensaje.

Por otra parte, para validar la segunda de las etapas, el investigador empleó la observación participante como método para describir comportamientos importantes de los estudiantes en situaciones de aprendizaje cotidianas. Además, el investigador registró 
pasajes significativos de la conducta y esfuerzos de los estudiantes en el contexto de las clases de Interpretación, para enunciar conclusiones acerca de la pertinencia o no de las acciones relacionadas con el desarrollo de la comprensión auditiva en función de la interpretación, derivadas de la etapa a evaluar.

Se realizaron un total de 18 observaciones, distribuidas en las asignaturas de Interpretación I, II, III, IV, V y VI. En todos los casos, el objetivo fundamental fue la evaluación de la materialización de las configuraciones y dimensiones del modelo didáctico para la comprensión auditiva en función de la interpretación.

Durante las observaciones, el autor de la investigación pudo realizar una descripción exacta y precisa de los acontecimientos específicos que tuvieron lugar en las clases observadas, así como describir las situaciones de aprendizaje de la habilidad auditiva lo suficientemente como para proporcionar significado a estos incidentes. De igual forma, se pudieron describir los acontecimientos que se relacionan con el desarrollo personal del estudiante y las interacciones con los demás sujetos que demanda la comprensión auditiva en función de la interpretación, dada la presencia de la re-expresión como un elemento importante dentro de la etapa post-auditiva.

Las principales interpretaciones o evaluaciones del autor de la investigación como parte de la implementación y la evaluación del modelo didáctico son las siguientes:

(a) Se pudo constatar que el reconocimiento de los elementos paralingüísticos de la comunicación (ritmo, entonación y acento del emisor del mensaje en el discurso oral) le permitió a los estudiantes mantener el registro, tono e intención comunicativa del mensaje oral comprendido en el momento de la re-expresión. Lo anterior está dado fundamentalmente porque se desarrollaron ejercicios de memoria que les permitieron descodificar y recodificar la información con la agilidad que requiere el proceso de interpretación, y por tanto, que el resultado final de la etapa post-auditiva fuera efectivo;

(b) Se observó el empleo de estrategias holísticas y atomísticas para la comprensión auditiva por parte de los estudiantes, con la adecuada guía y orientación del docente. Ello posibilitó el establecimiento de referencias sobre lo que se escucharía y el resumen de elementos para la contextualización de frases o palabras relacionadas con el contenido del mensaje, de modo que se facilitara la comprensión auditiva,

(c) Se llevó a cabo una adecuada orientación hacia la actividad auditiva por parte de los docentes, asegurándose que los estudiantes conocieran el objetivo de la tarea con anterioridad a la exposición al mensaje que sería comprendido. En ese sentido, se realizó más énfasis en las etapas de orientación realizadas en la asignatura Interpretación I y II,

(d) En el 87\% de las actividades se observaron tareas en las que la recodificación del mensaje debió realizarse del español al inglés y viceversa, lo que demuestra el carácter bilateral de la interpretación, y por ende, del proceso de comprensión auditiva.

En todos los casos, los docentes proporcionaron a los estudiantes oportunidades para personalizar le enseñanza-aprendizaje de la comprensión auditiva, en tanto potenciaron el establecimiento de relaciones entre la información que aportaron los textos orales y sus experiencias y vivencias personales. Esto permitió a los estudiantes comprender mejor el mensaje y transitar por cada una de las fases de la comprensión auditiva, así como las configuraciones que define el modelo didáctico propuesto.

De manera general, puede decirse que durante la puesta en práctica de las acciones correspondientes a la segunda etapa para la implementación y la evaluación del modelo didáctico, se demostró la pertinencia de las acciones propuestas para el tránsito por cada 
una de las configuraciones y la materialización de las dimensiones contenidas en el modelo didáctico, lo que permitió transformar el fin de la comprensión auditiva según lo previsto.

Asimismo, de estos análisis derivó la necesidad de introducir acciones relacionadas con el desarrollo de la comprensión auditiva en función de la interpretación desde disciplinas y años académicos precedentes al año académico donde se comienzan los estudios de interpretación como habilidad. Así, se rediseñaron acciones básicas que a un nivel más elemental pueden tenerse en cuenta para su aplicación en disciplinas correspondientes a años académicos precedentes.

La sistematización de la teoría en torno a la enseñanza-aprendizaje de la comprensión auditiva y la interpretación en la formación de intérpretes sirvió de base teórica para el establecimiento de las configuraciones del modelo didáctico que se presenta, así como para la fundamentación de sus dimensiones cognitivo-comunicativa, y sociocultural.

El estudio diagnóstico permitió comprobar las insuficiencias relacionadas con el desarrollo de la comprensión auditiva en función de la interpretación. Estas insuficiencias fueron constatadas en virtud de la aplicación de los instrumentos de investigación a estudiantes y profesores; lo que posibilitó determinar la solución a la problemática mediante el diseño e implementación de un modelo didáctico.

El modelo didáctico representa la dinámica de las relaciones entre sus configuraciones y dimensiones sobre la base de los aspectos cognitivo-comunicativos y socioculturales presentes en el discurso oral. Asimismo, se caracteriza por su carácter flexible, lo que permite su articulación en las asignaturas de interpretación en cualquiera de los años académicos en los que esta se imparte.

Los criterios de validez que desde el punto de vista teórico le otorgan los expertos a la concepción, configuraciones, dimensiones y acciones contenidas en las etapas para la implementación y la evaluación del modelo didáctico, son muy adecuados; según se pudo constatar durante la aplicación del Método Delphi.

La validez práctica del modelo didáctico se constató mediante la ejecución de las acciones correspondientes a las tres etapas para su implementación y evaluación, lo que permitió corroborar su efectividad para el desarrollo de la comprensión auditiva en función de la interpretación.

\section{REFERENCIAS BIBLIOGRÁFICAS}

Abreus González, A (2015). La comprensión auditiva en función de la interpretación en la formación del Licenciado en Lengua Inglesa con Segunda Lengua Extranjera [Tesis doctoral, Universidad de Cienfuegos].

Abreus, A. y Cima, D. (2013). De la teoría a la praxis en el uso de estrategias de traducción del inglés al español. Revista Ciencias Pedagógicas, (3). http://www.cienciaspedagogicas.rimed.cu

. (2016). La Traducción Oral a Simple Vista en los Licenciados en Lengua Inglesa. Apuntes didáctico-metodológicos. Revista Conrado, 2(54) http://conrado.ucf.edu.cu/index.php/RCr/ article/view/712

Abreus, A. (2019). Revisiting the teaching of listening comprehension to interpreters-to-be at the University of Cienfuegos. Una experiencia necesaria. Revista Conrado, 15(71), 116-121. https:// conrado.ucf.edu.cu/index.php/conrado/article/view/1183

Abreus, A. y Carballosa, A. M. (2014a). La enseñanza de la comprensión auditiva en inglés en función de la interpretación. Revista Universidad y Sociedad, 6(1), 31-39. http://rus.ucf.edu.cu/ index.php/rus/article/view/245 
(2014b). Modelo didáctico para el desarrollo de la habilidad de comprensión auditiva en función de la interpretación. Revista Universidad y Sociedad, 6(4), 33-38. http://rus.ucf.edu.cu/ index.php/rus/article/view/399

Abreus, A., y Carballosa González, A.M. (2015). Fundamentos para la enseñanza-aprendizaje de la comprensión auditiva en el marco de la interpretación. Revista Ciencias Pedagógicas, 2. http:// www.cienciaspedagogicas.rimed.cu

Casañ, J. C. (2009). Didáctica de las grabaciones audiovisuales para desarrollar la comprensión oral en el aula de lenguas extranjeras. MARCOELE, Revista de Didáctica Español como lengua extranjera, 9.

De Armas, N. y Valle, A. (2011). Resultados Científicos en la Investigación Educativa. Pueblo y Educación.

Gaiyan, W. (2010). Understanding Listening Comprehension Process and Effectively Organizing Listening Class. [Xi'an International Studies University].

Gimeno, J. (1981). Teoría de la enseñanza y desarrollo del currículo. Editorial ANAYA.

Güelmes, M. (2005). Modelo de cooperación interdisciplinaria para perfeccionar el desempeño del docente en la formación inicial del profesor general integral de secundaria básica. [Tesis doctoral, Universidad de La Habana].

Kornakov, P. K. (2000). Skills Interpreters Need. META, 45(2), 241-248.

Pöchhacker, F. (2010). The Role of research in interpreter education. Translation and Interpreting, 2(1). $1-10$.

Reinoso, C. (2007). Modelo pedagógico de actividad cooperada entre profesores generales integrales de secundaria básica. Editorial Academia.

Richards, J. C. y Burns, A. (2012). Tips for Teaching Listening: A Practical Approach. Pearson.

Rodríguez, G., Gil, J. y García, E. (2008). Metodología de la investigación cualitativa. Editorial Félix Varela.

Ruiz, A. (2003). La investigación educativa, operacionalización del problema. (Manuscrito sin publicar). Instituto Central de Ciencias Pedagógicas.

Siegel, J. (2015). Exploring Listening Strategy Instruction through Action Research. Palgrave McMillan.

Sierra, R. (2004). Modelo teórico para el diseño de una estrategia pedagógica en la educación primaria y secundaria básica [Tesis doctoral, Universidad de Ciencias Pedagógicas "Enrique José Varona"].

Sigarreta, J. M. (2001). Incidencia del tratamiento de los problemas matemáticos en la formación de valores [Tesis doctoral, Universidad de Holguín].

Vandergrift, L. y Goh, C. C. M. (2012). Teaching and Learning Second Language Listening: Metacognition in Action. Routledge. Taylor \& Francis. 
